\title{
Experimental Research on Preparation of Superfine Sand Concrete without Coarse Aggregate
}

\author{
Yubao Yang ${ }^{1, a}$, Yongjing Mao ${ }^{2, b}$, Yuepeng $\operatorname{Pan}^{3, c}$, Jie Chen ${ }^{4, d}$, Jie Leng ${ }^{5, e}$ \\ 1,2,3,4,5 College of harbor, coastal and offshore engineering, Hohai University, Nanjing, China \\ a809959529@qq.com, bmaomao19940405@126.com, '562167074@qq.com
}

\begin{abstract}
Keywords: superfine sand. method of semi-dry moulding. Concrete
Abstract: Using superfine sand as the main raw material and the superfine sand concrete without coarse aggregate was made by method of semi-dry moulding in this paper. The compressive, splitting tensile and immersion strength of the concrete material was studied to determine the influence of mix proportion of the binding materials. The results show that the optimal mixing ratio for materials used is $70 \%$ superfine sand, 15\% cement, 9\% ground granlated blastfurnace slag( GGBS), $2 \%$ gypsum and $4 \%$ lime. The compressive, tensile and immersion strength of this concrete at 28 days is $20 \mathrm{MPa}, 2.5 \mathrm{MPa}$ and $20 \mathrm{MPa}$ respectively, meeting the demand of civil engineering .In areas with an abundance of superfine sand but a shortage of ordinary aggregate, the material mentioned will have prominent economic value as well as promising application prospects.
\end{abstract}

\section{Instruction}

Superfine sand is a kind of fine sand with 0.7 1.5 fineness modulus. Its void content, soakage and silt content are relative high. Numerous researches have been taken on application of superfine sand. Shouchang Deng ${ }^{[1]}$ made concrete with superfine sand and found that the best dosage of this material is about 20\%. He also strictly controlled the water content for fear of segregation of concrete and decrease of its strength. Canyun $\mathrm{Yi}^{[2]}$ tried to improve the fine aggregate gradation by adding aggregate chips into superfine sand, which increased its fineness modulus. When the dosage of aggregate is half of the amount of fine aggregate, superfine sand concrete with high compressive strength $(22.3 \mathrm{MPa})$ can be produced. TU Er-hong. TU Er ${ }^{[3]}$ added superplasticizer, air entraining agent and fly ash to superfine sand concrete, by lowering the water-binder ratio and sand ratio, he finally prepared normal concrete with different strength levels as well as C20 pump concrete with high fluidity, good permeability and freezing resistance. Therefore, researches that using superfine sand as fine aggregate have been mature.

However, there are few studies on using superfine sand to produce concrete without coarse aggregate at present. Baochang Sun ${ }^{[4]}$ made some exploratory researches on this area. He produced such concrete by mixing cement, superfine sand and water. When the content of water is $420 \mathrm{~kg} / \mathrm{m}^{3}$, the compressive strength of such a concrete at 28 days peaked at $13.4 \mathrm{MPa}$. He also pointed that this kind of concrete is a new material in need of deeper researches. In China, there are large areas in short of medium and coarse sand. On the contrary, fine sand is widely distributed, mainly over Chongqing ${ }^{[5]}$, Liaohe and Northern Shaanxi ${ }^{[6]}$ regions. For areas like this, to prepare superfine sand concrete without coarse aggregate will have prominent economic value as well as promising application prospects.

In this paper, different ratios of cement, GGBS, gypsum and lime were mixed by semi-dry moulding with same dosage of superfine sand to produce concrete without coarse aggregate. Then the compressive, tensile and immersion strength of specimens at 28 days were tested and the optimal ratio of this concrete materials was determined. Finally, concrete with good comprehensive properties was produced whose compressive, tensile and immersion strength is above 20MPa, 2.5 $\mathrm{MPa}$ and $20 \mathrm{MPa}$ respectively, meeting the demand of civil engineering. 


\section{Experimental materials}

\subsection{Superfine sand}

The water content of superfine sand used in this experiment is about $5 \%$ and the $\mathrm{PH}$ value is 7.2 , which means this kind of sand is alkaline. As for its physical properties, particles of sand are isolated with no bond. Crystal particles are transparent with smooth edges and corners, contributing to bad plasticity and cohesion. The chemical composition of superfine sand is shown in Table 1 and its grain composition is shown in Table 2 .

It can be seen from Table 2 that the grain diameter of superfine sand ranges from $0.075 \mathrm{~mm}$ to $0.15 \mathrm{~mm}$, taking 95.4 percent of all particles, the fineness modulus of which is 0.82 .

Table 1. Chemical composition of superfine sand

\begin{tabular}{cccccccccc}
\hline Ingredients & $\mathrm{SiO}_{2}$ & $\mathrm{Al}_{2} \mathrm{O}_{3}$ & $\mathrm{Fe}_{2} \mathrm{O}_{3}$ & $\mathrm{CaO}$ & $\mathrm{MgO}$ & $\mathrm{K}_{2} \mathrm{O}$ & $\mathrm{Na}_{2} \mathrm{O}$ & $\mathrm{TiO}_{2}$ & $\mathrm{SO}_{3}$ \\
\hline Average value (\%) & 68.73 & 11.33 & 3.82 & 8.54 & 3.02 & 2.00 & 1.46 & 0.648 & 0.05 \\
\hline
\end{tabular}

Table 2. Grain composition of superfine sand

\begin{tabular}{ccccccc}
\hline Grain diameter (mm) & $1.18 \mathrm{~mm}$ & $0.6 \mathrm{~mm}$ & $0.3 \mathrm{~mm}$ & $0.15 \mathrm{~mm}$ & $0.075 \mathrm{~mm}$ & $<0.075 \mathrm{~mm}$ \\
\hline Mass percent (\%) & 0.02 & 0.04 & 2.99 & 76.06 & \multirow{2}{*}{19.34} & 1.29 \\
\hline
\end{tabular}

\subsection{Other materials}

Cement used in this experiment is $\mathrm{P} \bullet \mathrm{O} 42.5$ ordinary Portland cement.

GGBS used is produced by Nanjing Meibao new building materials Co, Ltd with standard of S95 and its chemical composition is shown in table 3.

Table 3. Chemical composition of GGBS

\begin{tabular}{ccccc}
\hline Ingredients & $\mathrm{SiO}_{2}$ & $\mathrm{Al}_{2} \mathrm{O}_{3}$ & $\mathrm{CaO}$ & $\mathrm{MgO}$ \\
\hline Average value (\%) & 32.9 & 15.36 & 37.04 & 10.52 \\
\hline
\end{tabular}

Lime involved is ordinary industrial grade with $96 \% \mathrm{CaO}$ content. And gypsum is Analytical reagent produced by Pharmaceutical Group Corporation.

\section{Experimental methods}

\subsection{Material preparation}

After being air dried, superfine sand was put into experiment directly

\subsection{Preparation and curing of specimens}

1. All materials were weighed precisely according to mix proportion then they were put into JJ-5 cement mortar mixer to be dry mixed for 2 minutes and wet mixed for 3 minutes successively.

2. The mixed materials were poured into $70.7 \mathrm{~mm} \times 70.7 \mathrm{~mm} \times 110 \mathrm{~mm}$ steel mold and made into specimens by vibration compression. After that, molds were put into SHT4305 universal testing machine and be pressed. The pressure on them increased to 20MPa with velocity of 400N/s and maintained until the specimens were produced.

3. Standard curing was taken in this experiment that specimens were put into standard curing room with temperature of $20^{\circ} \mathrm{C}$ and humidity of $95 \%$., after which properties of the cured specimens were tested.

\subsection{Test method}

The compressive and tensile strength of specimens were tested according to the standard of GB/T 50081-2002 (Standard for test method of mechanical properties on ordinary concrete). The immersion strength was tested accords to GB/T4111-1997 (Test methods for the small concrete hollow block and immersion strength is used as the indicator). The dry-shrinkage ratio, water 
absorption, volume density were tested accord to GB/T4111-1997 (Test methods for the small concrete hollow block).

\section{Experimental results and analysis}

\subsection{Optimum mix ratio of concrete materials}

\subsubsection{Influence of cement and GGBS's mixing ratio}

In this group of experiment, the content of superfine sand, lime and gypsum is $70 \%$, $3 \%$, and $4 \%$ respectively and the dosage of cement and GGBS is different. Then the influence of their mixing ratio can be observed. Experimental results are shown in Figure 3.

Figure 3 shows that, with the decrease of cement content and increase of GGBS dosage, the compressive strength of specimens declines slowly. The adding of GGBS can improve the tensile and immersion strengths of specimens: at the beginning, with GGBS adding, tensile and immersion strengths of specimens grow gradually, when their content is $14 \%$ and $9 \%$ respectively, the two strengths reach the maximum. But with GGBS's dosage continues to increase, the two strengths decrease on the contrary.

Because cement can effectively enhance strength of materials, its content plays a dominant role in concrete's strength. Also GGBS with good activity can not only improve concrete's cohesiveness and plasticity, but also affect its strength for pozzolanic effect. At the same time, $\mathrm{CaO}, \mathrm{SiO}_{2}$, and $\mathrm{Al}_{2} \mathrm{O}_{3}$ contained in GGBS can react with $\mathrm{Ca}(\mathrm{OH})_{2}$ and generate calcium silicate hydrate with lower basicity and calcium aluminate hydrate which can enhance the material. But when the content of GGBS continues to grow and cement shows a contrary direction, the basicity of concrete declines, resulting in weakness of gelation and bad mechanical properties of materials. Every fact taken into consideration, the optimum mixing ratio of cement and GGBS is $14 \%$ and $9 \%$ respectively.

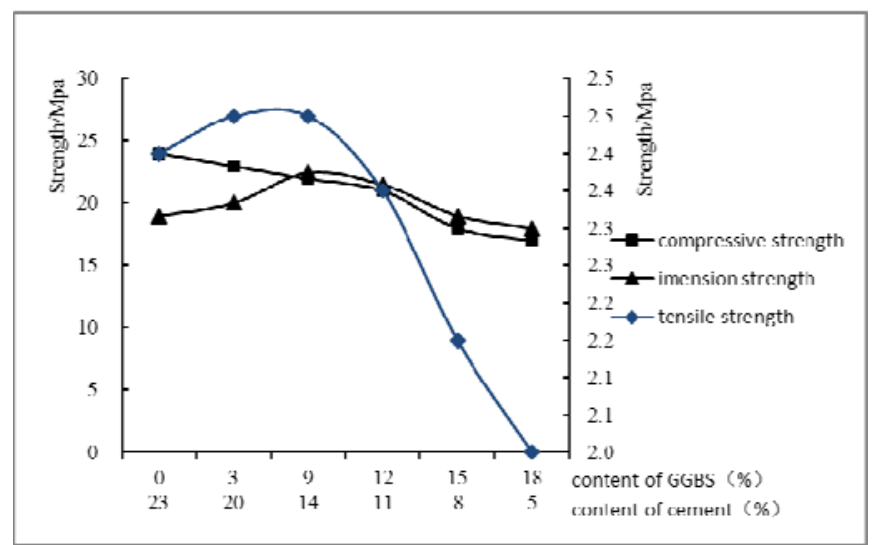

Figure 3. Influence of the missing ration of cement and GGBS on the performance of specimens

\subsubsection{Influence of GGBS and gypsum's mixing ratio}

In this experiment, the content of superfine sand, cement and lime is unchanged $(70 \%, 13 \%$, and $4 \%$ respectively) and the dosage of GGBS and gypsum is different, so the influence of their mixing ratio can be observed. Experimental result is shown in Figure 4.

Figure 4 illustrates that compressive, tensile and immersion strengths of specimens grow when more gypsum was added. When the content of gypsum is $2 \%$, all of the three strengths reach the peak (25.6MPa, 2.45MPa, and 25.6MPa respectively). However, when the dosage of gypsum continues to increase, mechanical properties of specimens decline and when its proportion larger than $4 \%$, the trend of decline is more distinct.

Reasons for this phenomena are as follows: Gypsum acts as activator in a concrete system without coarse aggregate and it can activate cement and GGBS. Also gypsum is able to react with calcium sulfoaluminate hydrates and generate ettringite, which can fill the interspace by expansion and effectively strengthen concrete. But when too much gypsum was added, overexpansion of 
ettringite can result in crack destruction of materials. Every fact taken into consideration, the optimum mixing ratio of GGBS and gypsum is $11 \%$ and $2 \%$ respectively.

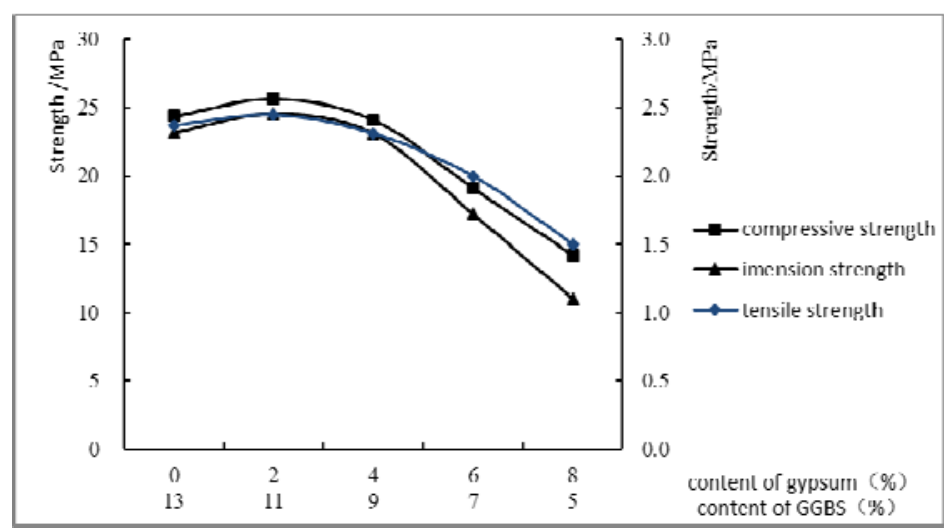

Figure 4. Influence of GGBS and gypsum's mixing ratio

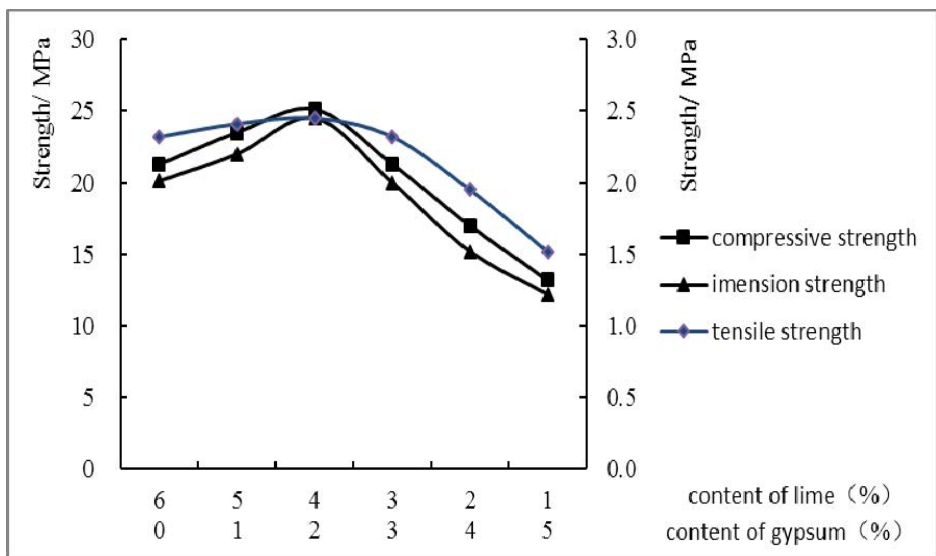

Figure 5. Influence of gypsum and lime's mixing ratio

\subsubsection{Influence of gypsum and lime's mixing ratio}

In this experiment, the content of superfine sand, cement and GGBS is unchanged $(70 \%, 14 \%$, and $10 \%$ respectively) and the dosage of lime and gypsum is different, so that the influence of their mixing ratio can be observed. Experimental result is shown in Figure 5.

Figure 5 demonstrates that, compressive, tensile and immersion strengths of specimens grow with the increase of lime. When its content is $4 \%$, mechanical properties of specimens reach peak and decline after that.

Lime is a kind of air hardening inorganic binding material, when mixed into materials, it experienced two processes namely crystallization and carbonation. Crystallization can generate calcium hydroxide with crystalline form, filling the interspace and strengthening concrete. During carbonation, $\mathrm{Ca}(\mathrm{OH})_{2}$ is likely to react with $\mathrm{CO}_{2}$ and generate $\mathrm{CaCO}_{3}$, which is insoluble in water and makes concrete harder. Reasons for the later decline can be divided into two aspects. Firstly, with the increase of cement, carbonation happens on the surface of concrete, forming compact carbon films and hindering $\mathrm{CO}_{2}$ from entering inside and the process of carbonation slows. Secondly, lime slaking consumes a large quantity of water, resulting in interspace after hardening and concrete weakens for this reason. Every factor taken into consideration, the optimum mixing ratio of lime and GGBS is $4 \%$ and $2 \%$ respectively.

As is analyzed before, the ratio of cement to GGBS is 14 to 9, GGBS to gypsum is 11 to 2 and gypsum to lime is 2 to 4 . So the ratio of cement, GGBS, gypsum and lime is $50 \%$ : $30 \%: 6.7 \%$ : $13.3 \%$. Considering the content of superfine sand is $70 \%$, the proportion of the four materials mentioned before is $15 \%, 9 \%, 2 \%$ and $4 \%$ respectively. 


\subsection{Test for physical mechanics of superfine sand concrete without coarse aggregate}

Concrete specimens were prepared according to optimum mixing ratio and were tested for compressive, tensile and immersion strength at 7, 28 and 56 days as well as dry-shrinkage ratio, water absorption and volume density. Test results are shown in Table 4.

Table 4. Physical mechanics of superfine sand concrete without coarse aggregate

\begin{tabular}{lccc}
\hline Curing days & 7d & 28d & 56d \\
\hline Compressive strength(MPa) & 11.42 & 20.35 & 25.10 \\
\hline Tensile strength(MPa) & 1.33 & 2.50 & 2.61 \\
\hline Immersion strength(MPa) & 13.27 & 24.25 & 26.40 \\
\hline Dry-shrinkage ratio (\%) & 0.009 & 0.01 & 0.02 \\
\hline Water absorption (\%) & 8.5 & 6.9 & 3.2 \\
\hline Volume density ((g/cm3) & 2.21 & 2.18 & 2.14 \\
\hline
\end{tabular}

It can be seen from Table 4 that this concrete has good early and later strength and its compressive, tensile and immersion strength at 28 days is $20 \mathrm{MPa}, 20 \mathrm{MPa}$ and 2.5MPa respectively. The dry-shrinkage ratio of this material is $0.01 \%$ which indicates less interspace and better durability. Water absorption is about $6.9 \%$ and decline with time, improving concrete's permeability resistance, frost resistance and erosion resistance. Volume density of this material is $2.18 \mathrm{~g} / \mathrm{cm}^{3}$, smaller than what of ordinary concrete. So construction made by this material has smaller self-weight and foundation stress, making itself safer.

\section{Conclusions}

1. Using superfine sand as the main raw material, the superfine sand concrete without coarse aggregate was made by method of semi-dry moulding. In this paper, superfine sand is main material and this material can be widely used in areas with abundant fine sand and limited coarse material.

2. When the content of superfine sand is $70 \%$ and percentage of cement, GGBS, gypsum and lime is $15 \%, 9 \%, 2 \%$ and $4 \%$ respectively, the compressive, tensile and immersion strength of this concrete at 28 days is $20 \mathrm{MPa}, 2.5 \mathrm{MPa}$ and $20 \mathrm{MPa}$ respectively. Also the concrete has good early and later strength as well as low dry-shrinkage ratio, water absorption and volume density. With good durability and combination property, this material has promising application prospects.

\section{References}

[1]Shouchang Deng, Yichun Ren, Kejian Li; Test research on fine-sand [J].Concrete,2004,173(3):43-44.

[2]Canyun Yi. Experimental research on concrete with superfine sand aggregate[J]. Zhejiang Hydrotechnics,1988,(2):33-35.

[3] TU Er-hong·TU Er-di 1,ZENG Li 2,LIU Zhi-dong 2,GAO Zhen. Superfine sand concrete mix designed test [J].Concrete, 2010,249(7):141-144.

[4]Baochang Sun. Superfine sand concrete without coarse aggregate [J]. Water Resources \& Hydropower of Northeast China,1991,83(5):2-5.

[5]Gelin Chen. Superfine Sand Pumping High-rise Building High-performance Concrete Construction Technology [J]. Chongqing Architecture, 2009, 8(12):31-33.

[6]Hongtang Wang.Performance experimental study on Shanbei desert's super-fine sand concrete[J]. Building Materials Science \& Technology,2002,33(1):30-31

[7]Qing Zheng.: The influences of dosages of slag powder on the mechanical property and durability of concrete [J]. China Concrete and Cement Products, 2011, (4):22-24.

[8]Tao Ji, Cai-Yi Chen, Yi-Zhou Zhuang, Jian-Feng Chen. A mix proportion design method of manufactured sand concrete based on minimum paste theory[J]. Construction and Building Materials, 2013,44:422-426. 